\title{
Inversion effects in the perception of the moving human form: A comparison of adolescents with autism spectrum disorder and typically developing adolescents
}

\author{
Laura Cleary', Kathy Looney', Nuala Brady' and Michael Fitzgerald²
}

\begin{abstract}
The "body inversion effect" refers to superior recognition of upright than inverted images of the human body and indicates typical configural processing. Previous research by Reed et al. using static images of the human body shows that people with autism fail to demonstrate this effect. Using a novel task in which adults, adolescents with autism, and typically developing adolescents judged whether walking stick figures-created from biological motion recordings and shown at seven orientations between $0^{\circ}$ and $180^{\circ}$ - were normal or distorted, this study shows clear effects of stimulus inversion. Reaction times and "inverse efficiency" increased with orientation for normal but not distorted walkers, and sensitivity declined with rotation from upright for all groups. Notably, the effect of stimulus inversion was equally detrimental to both groups of adolescents suggesting intact configural processing of the body in motion in autism spectrum disorder.
\end{abstract}

\section{Keywords}

autism spectrum disorders, biological motion perception, body inversion effect, configural processing

\section{Introduction}

Autism is a neurodevelopmental disorder characterized by impairments in social interaction, language, and communication and by atypical behaviors and interests (American Psychiatric Association (APA), 2006). In addition, a range of perceptual anomalies are reported in autism spectrum disorders (ASDs), which may precede or underlie problems in social cognition (Dakin and Frith, 2005).

The accurate perception of faces and biological motion is central to social cognition as faces and bodies convey information about others' behaviors, emotions, and intentions. While impaired perception of both faces and bodies is reported in ASD, recent reviews stress that the evidence for impairment is mixed and that the mechanisms underlying reported deficits are poorly understood (Simmons et al., 2009; Weigelt et al., 2012).

Configural processing - the integration of local elements or parts into a whole form - is characteristic of both face and body perception (Reed et al., 2006). As such, weak central coherence theory, which posits a processing bias for local at the expense of global form in ASD (Frith, 1989; Happé, 1999), provides a useful framework for thinking about anomalous perception of social stimuli. The theory receives support from demonstrations of superior local processing in ASD, as in the embedded figures and the Block Design tests (Jolliffe and Baron-Cohen, 1997; Shah and Frith, 1993). However, the idea that people with ASD are insensitive to configural information remains controversial (Mitchell and Ropar, 2004), and it has recently been argued that there is little to support the theory in the specific case of face perception (Weigelt et al., 2012).

In the study of face perception, "configuration" refers both to first-order relations between features - the generic arrangement of the eyes, nose, and mouth - and to secondorder relations which describe the precise geometric arrangement of the features in a face. Face inversion is known to disrupt configural processing, leading to slower response times and increased error rates in various perception, discrimination, and recognition tasks (Maurer et al.,

\footnotetext{
'University College Dublin, Ireland

${ }^{2}$ Trinity College Dublin, Ireland
}

Corresponding author:

Nuala Brady, School of Psychology, University College Dublin, Belfield, Dublin 4, Ireland.[AQ: 1]

Email: nuala.brady@ucd.ie 
2002). As these inversion effects are much greater for faces compared to other objects, the manipulation of stimulus orientation is a very useful tool in revealing stimulusspecific configural processing.

Bodies, like faces, are a class of stimuli with high selfsimilarity, being composed of a fixed set of parts that are arranged in a particular way (Reed et al., 2006). Therefore, and in analogy with face processing, expertise in discriminating and identifying bodies may involve an accurate representation of their shape or configuration. Research on the "body inversion effect" suggests that this is the case. Reed et al. (2003) first demonstrated that participants' ability to perceive differences in the posture of a pair of bodies is significantly better for upright than for inverted bodies, an effect not seen for other objects such as houses. A subsequent ERP study showed that the N170 to inverted bodies and inverted faces has a higher amplitude and longer latency than to upright bodies and faces, effects not seen for other objects (Stekelenburg and De Gelder, 2004). [AQ: 2] These inversion effects, at both behavioral and neural levels, suggest specialized configural processing of bodies.

Consistent with these findings, the perception of biological motion also shows marked inversion effects. In general, this research uses point-light displays (PLDs) animations made by recording the position of a small number of markers placed on the major joints of the body (Johansson, 1973) - that convey information about body configuration through the relative motion of local points of light. Despite the minimal information provided by PLDs, participants readily perceive the identity (Troje et al., 2005), gender (Barclay et al., 1978), and emotion (Dittrich et al., 1996) of the animations when these are presented in the normal, upright orientation.

However, participants find it harder to recognize inverted PLDs as human motion (Pavlova and Sokolov, 2000), to identify the actions portrayed (Sumi, 1984), and to tell the direction of movement (Troje and Westhoff, 2006). Infants as young as 4 months prefer to look at intact than scrambled PLDs when the stimuli are upright but show no preference when they are inverted (Fox and McDaniel, 1982). In adults, functional magnetic resonance imaging activation is stronger to upright than to inverted PLDs in posterior superior temporal sulcus, a region of the brain that is preferentially activated by biological motion (Grossman and Blake, 2001). In summary, the perception of human motion, like the perception of faces and static bodies, is especially vulnerable to stimulus inversion (Blake and Shiffrar, 2007). [AQ: 3]

To date, only one study has used inversion to investigate the perception of static bodies in ASD. In Reed et al. (2007), adult participants (10 ASD, 14 controls) viewed pairs of images of bodies which were identical or different in posture and indicated whether they were the same or different. They completed a similar task for images of faces. While the adults from the control group showed an inversion effect for both faces and bodies, those with ASD showed an inversion effect for faces but not for bodies.

While there is an extensive literature on the perception of biological motion in ASD (see Kaiser and Shiffrar (2009) and Pavlova (2012) for reviews), surprisingly few studies have measured inversion effects to study configural processing. In a recent eye tracking study, Klin et al. (2009) presented upright and inverted PLDs (animations of children's games) side by side in 2-year olds and measured looking times. While typically developing toddlers and toddlers with Down's syndrome spent significantly longer looking at the upright PLDs, those with ASD looked equally at upright and inverted displays. This suggests that biological motion is not particularly salient to young children with autism, a factor which could lead to atypical development of the brain regions involved in processing human movement.

Rutherford and Troje (2012) manipulated stimulus orientation in their study of configural processing of biological motion processing in adults $(n=14)$ with ASD. In both a detection task and a direction discrimination task, the adults with ASD were as sensitive as controls and their performance was similarly hindered by stimulus inversion. This lack of interaction between stimulus inversion and group (ASD/control) in a carefully designed psychophysical experiment is consistent with intact configural processing in ASD (see Weigelt et al., 2012).

This study investigates whether configural processing of the moving human form is impaired in ASD by measuring the effects of inversion in a novel task. We manipulated the appearance of a set of "stick figures"-stimuli created from PLDs of a walking person but with added form information-distorting them by local lengthening or shortening of the upper and lower limbs. These were presented at various orientations between upright and inverted to adolescents who were asked to say whether the figure was "normal" or "distorted." Since there are no existing data on this task, we conducted a pilot study with a neurotypical adult group to check that reaction times (RTs) and sensitivity varied as expected in the task and to gauge whether it would be suitable for younger participants. Specifically, we checked that error rates varied across orientation without ceiling or floor effects at upright and inverted orientations, respectively. We added form information to the PLDs to ensure that the distortions to the body configuration were still perceptible at inverted orientations.

Insofar as configural processing is compromised in ASD as suggested by weak central coherence theory, participants with ASD should show a weaker inversion effect than controls or the absence of an inversion effect as reported for static bodies by Reed et al. (2007). 


\section{Methods}

\section{Participants}

Adults (pilot study). A total of 13 University College Dublin (UCD) postgraduate students ( 8 females) with a mean age of 23.46 years ( $\mathrm{SD}=2.18$ years) volunteered to participate.

Adolescents. Participants for the experimental group were referred to the study by M.F., a Professor of Child and Adolescent Psychiatry whose research specialty is ASDs. Of 15 initially recruited, 1 participant who had a co-morbid diagnosis of attention deficit hyperactivity disorder performed at chance and their data were excluded. A second participant was asked to stop testing before the end of the study. The remaining 13, all males, ranged in age from 11 to 17 years, with a mean age of 13.93 years $(\mathrm{SD}=1.50$ years).

All were in full-time education, with 11 attending mainstream schools and 2 attending special schools for children with learning difficulties or autism. All 13 participants were diagnosed on the autism spectrum by the psychiatrist, who has over 25 years of clinical experience in the area of autism, using a structured assessment according to Diagnostic and Statistical Manual of Mental DisordersFourth Edition (DSM-IV) criteria (APA, 1994), with 11 diagnosed as high functioning, one as low functioning, and one as moderate functioning. [AQ: 4] Participants did not partake in a further research diagnosis using an instrument such as the Autism Diagnostic Intervention Schedule (ADOS; Lord et al., 1994). [AQ: 5] Research shows high agreement $(\sim 75 \%)$ between clinical diagnoses and diagnoses based on instruments such as the Autism Diagnostic Interview (ADI)/ADOS with inconsistencies largely reflecting false positives made by the research instruments (Mazefsky and Oswald, 2006).

As a measure of the magnitude of autistic traits, AutismSpectrum Quotient (AQ) scores were obtained for 12 of the 13 participants, their parents completing the AQ-Adolescent version (Baron-Cohen et al., 2006). ${ }^{1}$ [AQ: 6]Scores ranged from 23 to 48 with a mean score of $33.67(\mathrm{SD}=7.59)$. In Baron-Cohen et al. (2006), 86.8\% of a sample of adolescent boys with a clinical diagnosis of ASD and $0 \%$ of a control sample of adolescent boys scored at or above 30 .

Out of the 13 participants in the experimental group, 10 completed the Raven's Progressive Matrices (RPM), which measures analytical reasoning and fluid intelligence (Raven et al., 1998) and which is recommended as a short, nonverbal test of intelligence in autism (Bölte et al., 2009; Dawson et al., 2007). [AQ: 7] Scores ranged from 18 to 52 with a mean score of $41.5(\mathrm{SD}=12.37)$.

A total of 13 male control participants were recruited from a mainstream secondary school. They ranged in age from 12 to 15 years, with a mean age of 14.12 years $(\mathrm{SD}=$ 0.85 years). Their RPM scores ranged from 34 to 53 with a mean score of $45.46(\mathrm{SD}=5.30)$. The experimental and

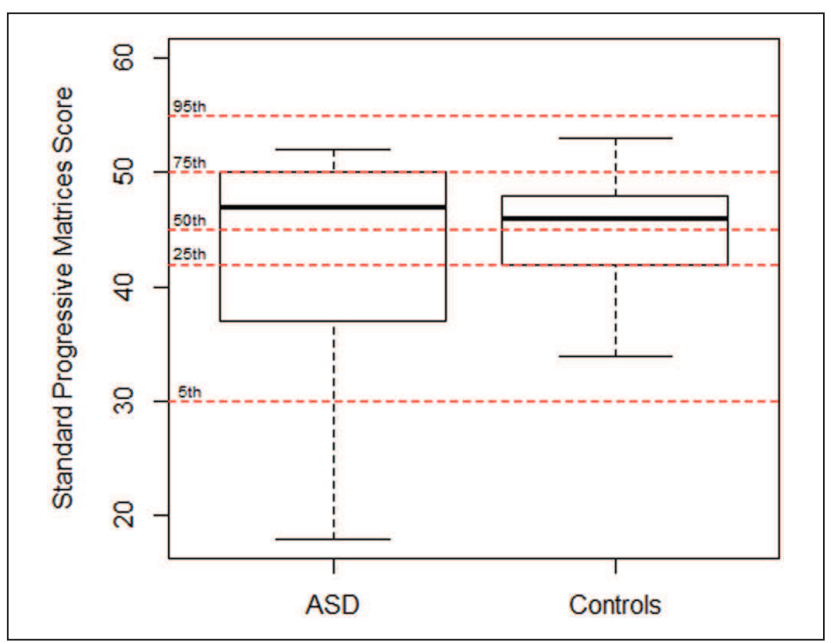

Figure I. Boxplots of the Raven's Standard Progressive Matrices scores for both groups showing the median by the thick horizontal line, the interquartile distance by the height of the box, and extreme values by the whiskers. The broken red lines crossing the graph show the 5th, 25th, 50th, 75th, and 95th percentiles as per norms for the British population; mean age $=$ 14 years (Raven et al., 1998). [AQ: 16]

control groups did not differ significantly in age, $t=-0.40$, $\mathrm{df}=28.97, p=0.69$, or in RPM scores, $t=-0.95$, df $=$ 11.55, $p=0.36$. Boxplots of RPM scores are plotted for both groups in Figure 1 where they are related to percentile scores for the general population. The ASD group shows more variability in IQ than the control group. All participants had normal or corrected-to-normal visual acuity. The study was approved by the UCD Human Research Ethics Committee and the Irish Health Services Executive Ethics Committee.

\section{Stimuli}

The biological motion stimuli were created from threedimensional (3D) coordinates of 13 markers positioned on the head, torso, and major joints of a person walking in position on a treadmill (Vanrie and Verfaillie, 2004). The original motion sequence was sampled at $30 \mathrm{~Hz}$ and was resampled here at $60 \mathrm{~Hz}$ and saved as a "stick figure" movie with the marker locations connected by lines (Figure 2). The stimuli consisted of forward-facing PLDs, both the original walker and a series of four "distorted" walkers. To prevent participants using a single local cue (e.g. shortened upper arm on right side of body) in detecting distortion on a given trial, four different types of distortion were introduced in MATLAB $^{\circledR}$. These included (a) elongating the torso and shortening the upper legs; (b) shortening the torso and elongating the upper legs; (c) shortening and elongating the upper and lower right arms and legs, respectively, while simultaneously elongating and shortening the upper and lower left arms and legs, respectively; and (d) making 


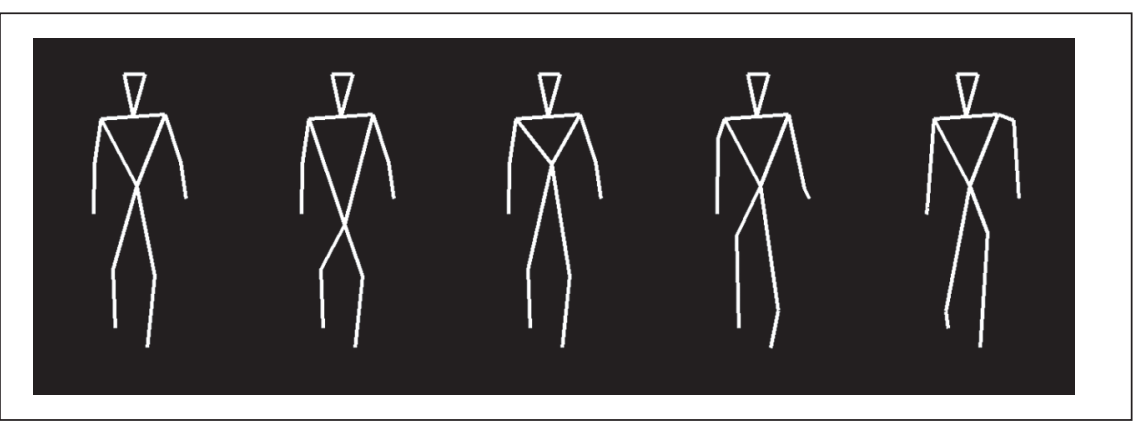

Figure 2. Static images from the movie clips depicting the five types of walkers in upright $\left(0^{\circ}\right)$ orientation. From left to right, these include the normal walker and four distorted walkers (see text for details), which are readily perceived as having deformities or abnormalities of gait when in motion.

the same distortions as in (c) but in mirror reversed form. A single static frame is shown for each of the walker types in Figure 2. The distortions are readily perceived as deformities or abnormalities of gait when in motion. All walkers were rotated clockwise between $0^{\circ}$ and $180^{\circ}$ in steps of $30^{\circ}$ for a total of seven orientations.

\section{Procedure}

The task was run on a Dell PC using Presentation ${ }^{\circledR}$. The display was run at $60 \mathrm{~Hz}$ with a spatial resolution of $1024 \times$ 768 pixels. The image subtended $\sim 11.9^{\circ}$ of visual angle at $\sim 50 \mathrm{~cm}$. Before a practice session of 30 trials, participants were presented with short movies of the normal and the four distorted walkers in upright orientation to acquaint them with the stimuli; all participants readily noticed the distortions.

Each participant completed 140 experimental trials, 70 normal walker and 70 distorted walker trials, each batch of 70 trials being made up of 10 trials at each of the seven orientations. The 70 "distorted trials" were made up of 21 trials using distorted walker 1, 21 trials using distorted walker 2, and 14 trials each using distorted walkers 3 and 4 . These ratios were used as the distortions present in distorted walkers 3 and 4 are similar, the walkers being mirror images of each other. The 140 trials were presented in pseudo-random order. Participants were asked to respond as quickly as possible on each trial by pressing one of two keys, using their right and left index finders to indicate "normal" and "distorted," respectively, once they were reasonably sure that they perceived a normal or distorted walker.

\section{Statistical analyses}

RT data were analyzed in R (R Development Core Team, 2010) using analysis of variance (ANOVA) with withinsubject factors of Angle (seven levels) and Condition (Normal/Distorted). Greenhouse-Geisser epsilon ( $\varepsilon$ ) corrections are reported when Mauchly's test for sphericity was significant and effect sizes are given by generalized eta squared $\left(\eta_{G}^{2}\right)$ (Bakeman, 2005). Two further measures of performance, sensitivity $\left(d^{\prime}\right)$ and "inverse efficiency," were calculated at two composite angles of rotation, "upright" (combining data from $0^{\circ}, 30^{\circ}$, and $60^{\circ}$ ) and "inverted" (combining data from $120^{\circ}, 150^{\circ}$, and $180^{\circ}$ ); combining individual participant's data across conditions increases the accuracy of $d^{\prime}$ estimates (Macmillan and Creelman, 1991: ch. 11). As previously used in ASD research (e.g. Falter and Bailey, 2011), "inverse efficiency" controls for speed-accuracy trade-offs and was calculated as follows: for each participant and condition the mean RT for correct trials was divided by the proportion of correct responses in that condition.

\section{Results}

\section{Adults (pilot study)}

Figure 3(a) plots mean RT for correct trials only (79.67\% of normal and $82.96 \%$ of distorted) by angle to say that a walker was normal or distorted. RTs are similar in the two conditions for upright walkers $\left(0^{\circ}\right)$ but then diverge, increasing gradually for the normal walkers out to $120^{\circ}$, whereas RTs are more stable across rotation for the distorted walkers.

ANOVA showed significant main effects of Condition, $F(1,12)=7.41, p<0.05, \eta_{G}^{2}=0.033$, and Angle, $F(6,72)=$ 5.15, $p<0.01, \eta_{G}^{2}=0.033$, and a significant Condition $\times$ Angle interaction, $F(6,72)=3.14, p<0.01, \eta_{G}^{2}=0.023$. Post hoc analyses showed that the effect of Angle was highly significant in the normal walker condition, $F(6,72)=$ 5.08, $p<0.01, \eta_{G}^{2}=0.07$, but was not significant in the distorted walker condition, $F(6,72)=2.02, p=0.13, \varepsilon=$ $0.50, \eta_{G}^{2}=0.024$.

Figure 3(b) plots inverse efficiency for composite upright and inverted orientations. Efficiency shows a marked decline in performance across orientation for the normal but not distorted walkers. ANOVA showed a significant main effects of Angle, $F(1,12)=12.47, p<0.01$, 


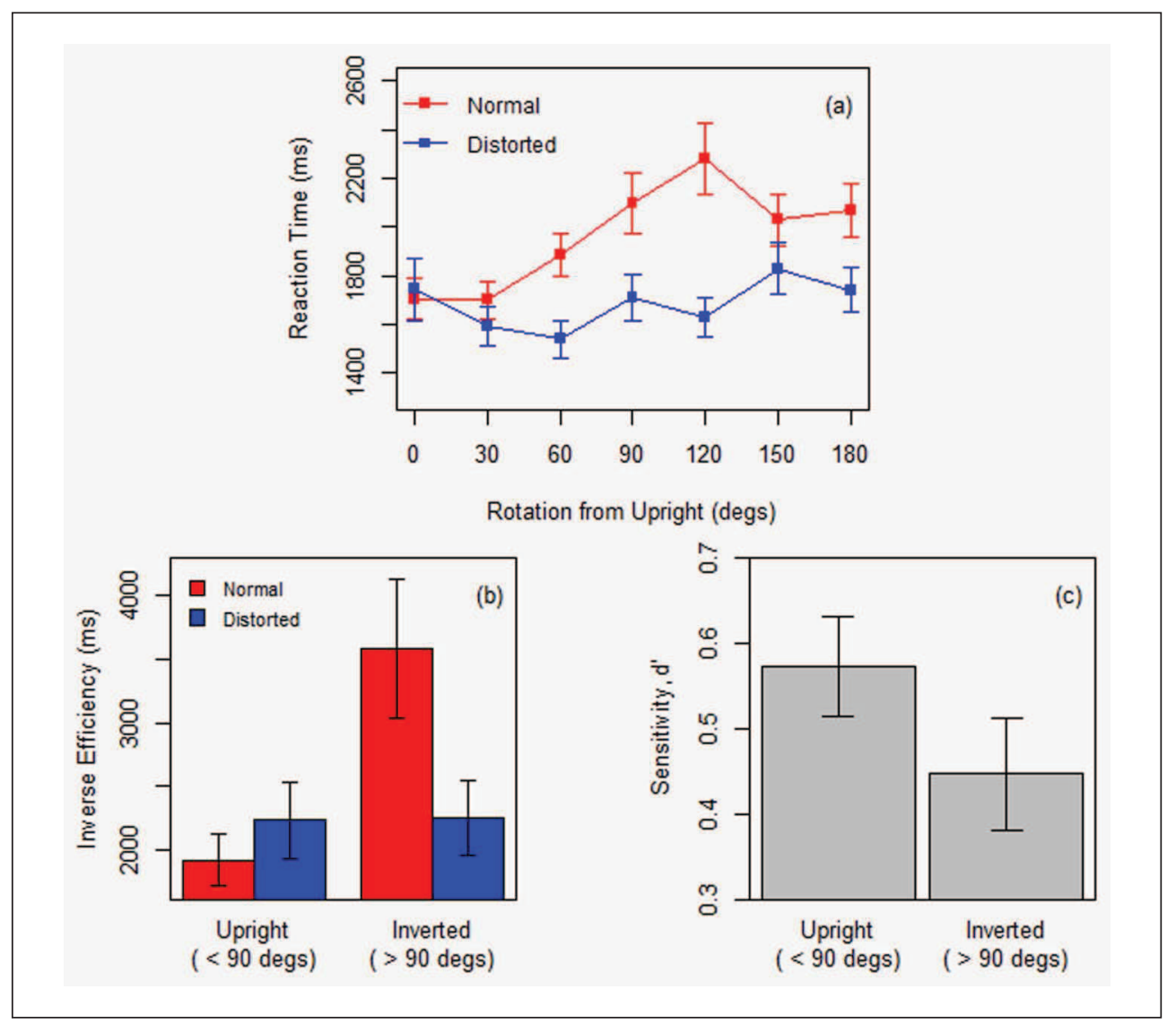

Figure 3. Adult pilot data: (a) Reaction time is plotted as a function of angle of rotation for both the normal (red) and distorted (blue) walkers. (b) Inverse efficiency, split by normal (red) and distorted (blue) conditions, and (c) sensitivity is plotted as a function of "upright" and "inverted" angles. Error bars show \pm I SEM. $d$ ' is scaled so that maximum sensitivity is I.0.

$\eta_{G}^{2}=0.10$, and a significant Condition $\times$ Angle interaction, $F(1,12)=10.57, p<0.01, \eta_{G}^{2}=0.098$. Post hoc analyses showed that the effect of Angle was highly significant in the normal walker condition, $F(1,12)=12.88, p<0.01$, $\eta_{G}^{2}=0.25$, but was not significant in the distorted walker condition, $F(1,12)=0.01, p=0.93, \eta_{G}^{2} \sim 0.078$. Figure 3 (c) plots $d^{\prime}$ at the two composite angles and shows a clear decrease in sensitivity from upright to inverted angles of rotation, which is significant, $F(1,12)=8.75, p<0.05$, $\eta_{G}^{2}=0.078$.

\section{Adolescents, ASD, and controls}

Reaction times. For the adolescent ASD (control) data, $71.32 \%(75.71 \%)$ of normal and $75.38 \%(80.42 \%)$ of distorted walker trials were correct. Exploratory data analyses highlighted a small number of outliers, and $0.45 \%(1.13 \%)$ of correct trials for the ASD (control) group were removed.

Plots of mean RT by walker orientation are shown for the ASD and control groups in Figure 4(a) and (b), respectively. Both plots share the overall characteristics of the adult data, in that RTs in the normal walker condition increase gradually with angle of rotation and then decline again, whereas RTs are more stable across rotation from the upright for the distorted walkers.

ANOVA showed a significant Angle $\times$ Condition interaction only, $F(6,144)=5.92, p<0.01, \eta_{G}^{2}=0.023$. The main effect of Group was not significant $(F(1,24)=0.95, p=$ 0.34 ), neither of the two-way interactions involving Group was significant (with Angle, $F(6,144)=1.12, p=0.35$, with Condition, $F(1,24)=0.98, p=0.33)$, and the three-way interaction, Group $\times$ Angle $\times$ Condition, was not significant $(F(6,144)=0.76, p=0.61)$. Post hoc analyses showed that the effect of Angle was highly significant in the normal walker condition, $F(6,150)=5.18, p<0.01, \varepsilon=0.66$, $\eta_{G}^{2}=0.06$, but was not significant in the distorted walker condition, $F(6,150)=1.27, p=0.29, \varepsilon=0.73, \eta_{G}^{2}=0.009$, mirroring the results found with adult participants.

Figure 5 plots inverse efficiency (upper panel) and sensitivity (lower panel) at composite "upright" and "inverted" angles for both ASD and control participants. As with the adult data, the inverse efficiency plots showed a marked dip in performance with inversion for the normal but not distorted walkers. ANOVA showed a significant Angle $\times$ Condition interaction, $F(1,24)=8.48, p<0.01, \eta_{G}^{2}=0.08$. 


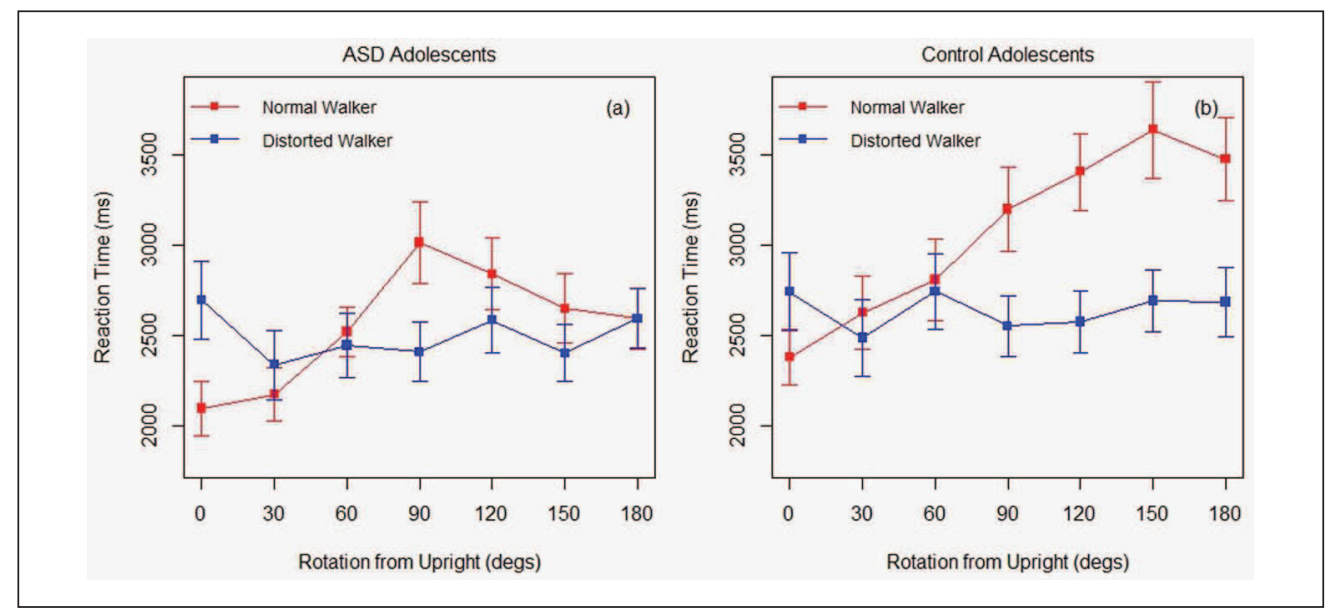

Figure 4. Reaction time is plotted as a function of angle of rotation from upright for both the normal (red) and distorted (blue) walkers for (a) the adolescent autism spectrum disorder participants and (b) the adolescent control participants. Error bars show \pm I SEM.

Post hoc tests showed that the effect of Angle was significant in both the normal, $F(1,25)=7.96, p<0.01, \eta_{G}^{2}=0.13$, and in the distorted, $F(1,25)=5.64, p=0.03, \eta_{G}^{2}=0.02$, walker conditions, but with inverse efficiency increasing with inversion in the normal while decreasing somewhat in the distorted walker condition (Figure 5). The main effect of Group was not significant $(F(1,24)=0.19, p=0.66)$, neither of the two-way interactions involving Group was significant (with Angle, $F(1,24)=0.19, p=0.66$, with Condition, $(F(1,24)=0.02, p=0.89)$, and the three-way interaction, Group $\times$ Angle $\times$ Condition, was not significant $(F(1,24)=0.04, p=0.84)$.

While sensitivity (lower panel, Figure 5) does decrease from upright to inverted angles for both groups, the difference is more marked for the control group. ANOVA with a between-subject factor of Group and within-subject factors of Angle showed a significant effect of Angle, $F(1,24)=$ $8.71, p<0.01, \eta_{G}^{2}=0.02$. Neither the main effect of Group $(F(1,24)=0.44, p=0.51)$ nor the Group $\times$ Angle interaction $(F(1,24)=1.85, p=0.19)$ was significant.

Finally, for the ASD group, Pearson's correlation between participants' AQ scores and their sensitivity scores was not significant in either the upright, $t(10)=-0.18, p=$ 0.86 , or the inverted, $t(10)=0.09, p=0.93$, conditions.

\section{Discussion}

This study compared adolescents with ASD and typically developing adolescents' ability to discriminate between normal and distorted moving stick figures at orientations ranging from upright to upside-down. Successful completion of the task likely involves a discrimination of body "configuration," a type of processing that operates best at upright orientations of the body and which may be anomalous in ASD (Reed et al., 2007).

The pilot study with adult participants shows that when form information is added to PLDs to make stick figures that move like real people, the perception of distortion is readily perceived for upright stimuli. While participants responded rapidly and with high accuracy to say whether the figures were normal or distorted at $0^{\circ}$, their capacity to discriminate declined as the figures were rotated from the upright. First, there was a marked decrease in sensitivity for the task as the figures were rotated away from the upright toward the inverted. Second, RT to correctly identify the normal walker gradually increased as it was rotated away from $0^{\circ}$, whereas RT to correctly identify the distorted walkers remained more constant across orientation.

What accounts for this difference in RT profile across conditions? The usual explanation for the inversion effect is that configural processing is tied to orientations at or near the upright and that participants rely on more "local cues" when processing inverted stimuli. RT increases gradually with angle of rotation in the normal condition suggesting a gradual shift from global to more local processing as previously reported in the face perception literature; for example, in a study of the Thatcher illusion, Lewis (2001) showed that RT to identify an undistorted face as normal increased gradually with rotation from upright. However, in the distorted walker condition RT is more constant across angle of rotation. It is possibly that participants shifted more rapidly to using "local cues" to distortion which are more readily available in the distorted walker condition. While the definition of local, feature-based processing and configural processing is well defined in the case of face perception, this is not so for bodies. Reed et al. (2006) discuss the particular utility of Marr's definition of "structural information" which refers both to the organization of parts relative to the whole body and to the organization of parts relative to each other. It is very likely that participants are using such local structural (or local configural) cues in rapidly picking up on distortion at inverted orientations, for example, shortened or lengthened torso relative to head, shortened or lengthened lower arm, or leg relative to upper 


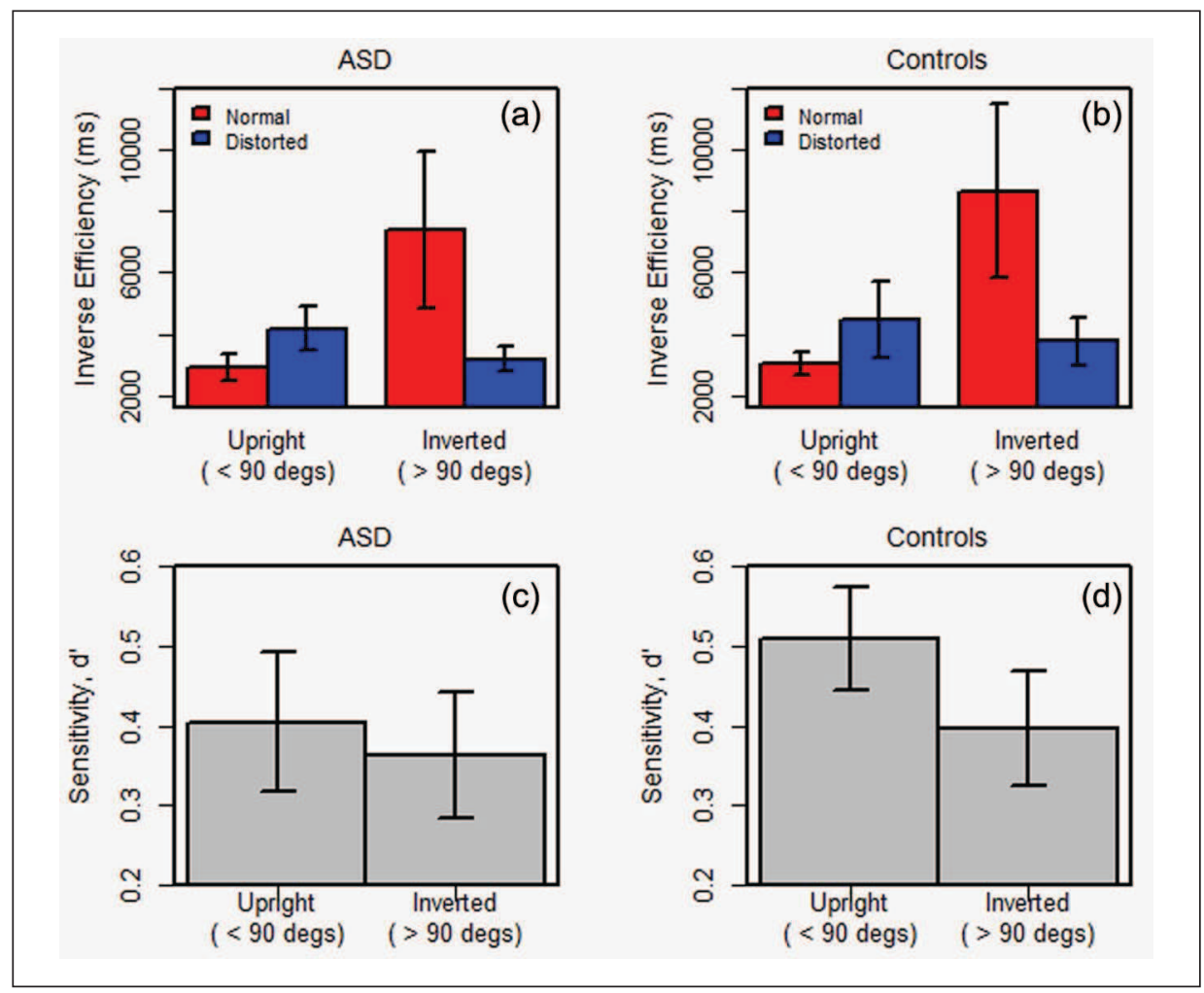

Figure 5. Inverse efficiency, split by normal (red) and distorted (blue) conditions, is shown for the (a) ASD and (b) control adolescents for composite "upright" and "inverted" angles. Sensitivity is similarly plotted for the (c) ASD and (d) control groups. Error bars show \pm I SEM. $d^{\prime}$ is scaled so that maximum sensitivity is I.0. ASD: autism spectrum disorder.

arm or leg (see Figure 2). Our own impression in viewing the stimuli is of focusing on these relatively "local structural cues" for the inverted walkers and on the whole body for the upright walkers which appear to be deformed, limping, and so on.

The finding of an inversion effect in our task is consistent with research using more conventional PLDs. For example, Pavlova and Sokolov (2000) showed that sensitivity to the dynamics of PLDs is orientation dependent across a range of orientations centered on the upright. And there is a large body of research showing that inversion disrupts detection of PLDs in noise (e.g. Rutherford and Troje, 2012) and the ability to determine higher level properties such as walker gender (Barclay et al., 1978) and emotion as expressed through body movement (Dittrich et al., 1996).

The results of the main study with adolescents show a similar pattern to that of the adult study in that there was a significant effect of orientation on RT for the normal walker, but not for the distorted walker. This effect did not differ between the groups so that both the ASD and control groups showed a slowing of RT as the normal walker was rotated from upright but fairly constant RTs across changes in orientation for the distorted walkers. Although the two groups show a somewhat different RT by orientation profile in the normal walker condition (Figure 4), statistically, the
Group $\times$ Orientation $\times$ Condition interaction was not significant.

Analysis of inverse efficiency data, collapsed across composite upright and inverted orientations, similarly showed a significant effect of orientation but a non-significant effect of group and, particularly, a non-significant group by orientation interaction. This indicates that the inversion effect was present and comparably for both groups, suggesting intact configural processing in those with ASD. Overall, sensitivity was significantly higher for upright orientations than for inverted orientations. Again, there were no significant effects of group membership on sensitivity in the main analysis, although we note that the trend in Figure 5 (lower panel) is toward a greater difference in sensitivity with orientation for the controls and we acknowledge the small sample size as a limitation of our study.

In summary, these results suggest that adolescents with ASD, like typically developing adolescents, process upright point light walkers configurally and shift to using more local processing as the figures were rotated away from $0^{\circ}$. While these results are inconsistent with a strong version of the weak central coherence theory which suggest that people with ASD have a natural tendency to use local over global cues (Frith, 1989; Happé, 1999), they are consistent 
with revisions of the theory which suggest that global cues may be used when they are particularly useful to the task (Happé and Frith, 2006).

While the results of this study argue for an inversion effect and intact configural processing in the perception of bodies in ASD, one previous study using static images of bodies finds differently (Reed et al., 2007). There are obvious differences between the tasks. First, this study uses dynamic stick figures that are derived from PLDs of a person in motion and a task that involves detecting distortion, whereas Reed et al. (2007) use static images of real bodies and a same/different paradigm. It is likely that the addition of biological movement enhances the perception of global form in our study. Consistent with this, both typically developing children and children with ASD have been shown to respond faster, although with similar accuracy, in judging whether two people are interacting in a social or non-social way when dynamic stick figures rather than conventional PLDs were used (Centelles et al., 2012).

Our results are consistent with a number of studies which use conventional PLDs, rather than stick figures, and which show comparable performance between typically developing and ASD groups. These include reports of comparable performance in identifying people and actions in PLDs (e.g. Hubert et al., 2007; Moore et al., 1997; Parron et al., 2008), comparable use of configural information in determining the direction of moving point light walkers (Murphy et al., 2009), and comparable thresholds for the detection of biological motion (Saygin et al., 2010). Notable for including a study of the effects of stimulus inversion is the study by Rutherford and Troje (2012), who report no differences in psychophysical thresholds for adults with ASD and a control group and similar inversion effects for both groups.

There are, however, numerous reports of performance differences on biological motion tasks between children and adolescents with ASD and age- and IQ-matched controls (e.g. Annaz et al., 2010; Blake et al., 2003; Kaiser et al., 2010; Koldewyn et al., 2010; Price et al., 2012) with further reports that adults with ASD may rely on different neural processing to perform biological motion tasks even when their behavioral performance is comparable to that of controls (Freitag et al., 2008; McKay et al., 2012). Thus, the age of testing may be an important determinant of whether researchers find significant differences between groups in their perception of biological motion, as children with ASD may take longer to develop these skills or may use different strategies as adults to solve the experimental tasks. Increasingly, the importance of IQ is also stressed as a relevant factor in whether group differences are found, for example, testing a very large sample of young people with ASD $(n=89)$, Jones et al. (2011) report no impairment in the perception of biological motion except in a subsample of participants (18\%) with low IQ.
While some studies of biological motion processing in ASD have shown that the extent of autistic traits is predictive of performance, with higher symptom scores leading to poorer performance (e.g. Blake et al., 2003), others have not (e.g. Rutherford and Troje, 2012). While we found that AQ scores were not predictive of sensitivity on our task, we acknowledge the small sample size as a limitation of the study; replication with a much larger sample size would allow for a more rigorous comparison of participants with different scores on questionnaires such as the AQ or on research instruments such as the ADI/ADOS.

Returning to the central question of this article, our study joins others in showing that configural information, important to the discrimination of human posture and movement, is available to people with ASD (Murphy et al., 2009; Rutherford and Troje, 2012). Configuration is also important to face perception and recognition. Two studies have shown spontaneous configural processing in people with ASD for face stimuli using the Thatcher illusion (Rigby et al., 2009; Rouse et al., 2004). [AQ: 8] And, in their recent review of face processing in autism, Weigelt et al. (2012) conclude that there is little evidence that people with autism process faces in a qualitatively different way than people without autism and, in particular, stress that they demonstrate intact configural processing.

In conclusion, using a novel task in which participants judge whether dynamic stick figures, created from point light recordings of a person walking, are distorted or not, we find that adolescents with ASD show comparable performance to age- and IQ-matched controls.

\section{Acknowledgements}

We would like to thank all the participants and their parents for their time and effort.

\section{Funding}

This research received no specific grant from any funding agency in the public, commercial, or not-for-profit sectors. [AQ: 9]

\section{Note}

1. Although one participant fell outside the age range for the Adolescent Autism Quotient (> 16 years), his parent completed the questionnaire on his behalf as he had learning difficulties.

\section{References}

American Psychiatric Association (APA) (2006) Diagnostic and Statistical Manual of Mental Disorders (DSM-IV-TR). 4th ed. text rev. Washington, DC: APA.

Annaz D, Remington A, Milne E, et al. (2010) Development of motion processing in children with autism. Developmental Science 13(6): 826-838. 
Bakeman R (2005) Recommended effect size statistics for repeated measures designs. Behavior Research Methods 37: 379-384.

Barclay CD, Cutting JE and Kozlowski LT (1978) Temporal and spatial factors in gait perception that influence gender recognition. Perception \& Psychophysics 23: 145-152.

Baron-Cohen S, Hoekstra R, Knickmeyer R, et al. (2006) The autism-spectrum quotient (AQ)-adolescent version. Journal of Autism and Developmental Disorders 36(3): 343-350.

Blake R and Shiffrar M (2007) Perception of human motion. Annual Review of Psychology 58: 47-73.

Blake R, Turner L, Smoski M, et al. (2003) Visual recognition of biological motion is impaired in children with autism. Psychological Science 14(2): 151-157.

Bölte S, Dziobek I and Poustka F (2009) Brief report: the level and nature of autistic intelligence revisited. Journal of Autism and Developmental Disorders 39(4): 678-682.

Centelles L, Assaiante C, Etchegoyhen K, et al. (2012) From action to interaction: exploring the contribution of body motion cues to social understanding in typical development and in autism spectrum disorders. Journal of Autism and Developmental Disorders 43: 1140-1150. [AQ: 10]

Dakin S and Frith U (2005) Vagaries of visual perception in autism. Neuron 48(3): 497-507.

Dawson M, Soulières I, Gernsbacher MA, et al. (2007) The level and nature of autistic intelligence. Psychological Science 18(8): 657-662.

Dittrich WH, Troscianko T, Lea SEG, et al. (1996) Perception of emotion from dynamic point-light displays represented in dance. Perception 25: 727-738.

Falter CM and Bailey AJ (2011) Perception of mirror symmetry in autism spectrum disorders. Autism 16(6): 622-626. [AQ: 11]

Fox R and McDaniel C (1982) The perception of biological motion by human infants. Science 218: 486-487.

Freitag C, Konrad C, Häberlen M, et al. (2008) Perception of biological motion in autism spectrum disorders. Neuropsychologia 46: 1480-1494.

Frith U (1989) Autism: Explain the Enigma. Oxford: Basil Blackwell.

Grossman ED and Blake R (2001) Brain activity evoked by inverted and imagined biological motion. Vision Research 41: $1475-1482$.

Happé F (1999) Autism: cognitive deficit or cognitive style? Trends in Cognitive Sciences 3(6): 216-222.

Happé F and Frith U (2006) The weak coherence account: detailfocused cognitive style in autism spectrum disorders. Journal of Autism and Developmental Disorders 36(1): 5-25.

Hubert B, Wicker B, Moore D, et al. (2007) Brief report: recognition of emotional and non-emotional biological motion in individuals with autistic spectrum disorders. Journal of Autism and Developmental Disorders 37(7): 1386-1392.

Johansson G (1973) Visual perception of biological motion and a model for its analysis. Perception \& Psychophysics 14(2): 201-211.

Jolliffe T and Baron-Cohen S (1997) Are people with autism and Asperger syndrome faster than normal on the embedded figure test? Journal of Child Psychology and Psychiatry and Allied Disciplines 38: 527-534. [AQ: 12]

Jones CRG, Swettenham J, Charman T, et al. (2011) No evidence for a fundamental visual motion processing deficit in adolescents with autism spectrum disorders. Autism Research 4: $347-357$
Kaiser M and Shiffrar M (2009) The visual perception of motion by observers with autism spectrum disorders: a review and synthesis. Psychonomic Bulletin \& Review 16(5): 761-777.

Kaiser M, Delmolino L, Tanaka J, et al. (2010) Comparison of visual sensitivity to human and object motion in autism spectrum disorder. Autism Research 3(4): 191-195.

Klin A, Lin DJ, Gorrindo P, et al. (2009) Two-year-olds with autism orient to non-social contingencies rather than biological motion. Nature 459: 257-261.

Koldewyn K, Whitney D and Rivera S (2010) The psychophysics of visual motion and global form processing in autism. Brain 133: 599-610.

Lewis M (2001) The lady's not for turning: rotation of the Thatcher illusion. Perception 30: 769-774.

Lord C, Rutter M and Couteur A (1994) Autism diagnostic interview-revised: a revised version of a diagnostic interview for caregivers of individuals with possible pervasive developmental disorders. Journal of Autism and Developmental Disorders 24(5): 659-685.

McKay L, Simmons D, McAleer P, et al. (2012) Do distinct atypical cortical networks process biological motion information in adults with autism spectrum disorders? Neuroimage 59: 1524-1533.

Macmillan NA and Creelman CD (1991) Signal Detection Theory: A User's Guide, ch. 1. Cambridge: Cambridge University Press.

Maurer D, Grand RL and Mondloch CJ (2002) The many faces of configural processing. Trends in Cognitive Sciences 6(6): 255-260.

Mazefsky CA and Oswald DP (2006) The discriminative ability and diagnostic utility of the ADOS-G, ADI-R, and GARS for children in a clinical setting. Autism 10(6): 533-549.

Mitchell P and Ropar D (2004) Visuo-spatial abilities in autism: a review. Infant and Child Development 13: 185-198.

Moore D, Hobson P and Lee A (1997) Components of person perception: an investigation with autistic, non-autistic retarded and typically developing children and adolescents. British Journal of Developmental Psychology 15(4): 401-423.

Murphy P, Brady N, Fitzgerald M, et al. (2009) No evidence for impaired perception of biological motion in adults with autistic spectrum disorders. Neuropsychologia 47: 3225-3235.

Parron C, Da Fonseca D, Santos A, et al. (2008) Recognition of biological motion in children with autistic spectrum disorders. Autism 12(3): 261-274.

Pavlova M and Sokolov A (2000) Orientation specificity in biological motion perception. Perception \& Psychophysics 62(5): 889-899.

Pavlova MA (2012) Biological motion processing as a hallmark of social cognition. Cerebral Cortex 22(5): 981-995.

Price KJ, Shiffrar M and Kerns KA (2012) Movement perception and movement production in Asperger's syndrome. Research in Autism Spectrum Disorders 6(1): 391-398.

Raven J, Raven JC and Court JH (1998) Manual for Raven's Progressive Matrices and Vocabulary Scales. San Antonio, TX: Harcourt Assessment.

R Development Core Team (2010) R: A Language and Environment for Statistical Computing. Vienna: R Foundation for Statistical Computing. Available at: http://www.R-project.org

Reed C, Beall P, Stone V, et al. (2007) Brief report: perception of body posture-what individuals with autism spectrum 
disorder might be missing. Journal of Autism and Developmental Disorders 37(8): 1576-1584.

Reed CL, Stone VE, Bozova S, et al. (2003) The body-inversion effect. Psychological Science 14: 302-308.

Reed CL, Stone VE, Grubb JD, et al. (2006) Turning configural processing upside down: part and whole body postures. Journal of Experimental Psychology: Human Perception and Performance 32(1): 73-87. [AQ: 13]

Rutherford MD and Troje NF (2012) IQ predicts biological motion perception in autism spectrum disorders. Journal of Autism and Developmental Disorders 42: 557-565.

Saygin A, Cook J and Blakemore S (2010) Unaffected perceptual thresholds for biological and non-biological form-frommotion perception in autism spectrum conditions. PLoS One 5(10): e13491.

Shah A and Frith U (1993) Why do autistic individuals show superior performance on the block design task? Journal of Child Psychology and Psychiatry and Allied Disciplines 34(8): 1351-1364. [AQ: 14]
Simmons DR, Robertson AE, McKay LS, et al. (2009) Vision in autism spectrum disorders. Vision Research 49: 2705-2739.

Stekelenburg JJ and De Gelder B (2004) The neural correlates of perceiving human bodies: an ERP study on the body-inversion effect. Neuroreport 15(5): 777-780.

Sumi S (1984) Upside-down presentation of the Johansson moving light-spot pattern. Perception 13: 283-286.

Troje NF and Westhoff C (2006) The inversion effect in biological motion perception: evidence for a "life detector"? Current Biology 16: 821-824.

Troje N, Westhoff C and Lavrov M (2005) Person identification from biological motion: effects of structural and kinematic cues. Perception \& Psychophysics 67(4): 667-675.

Vanrie J and Verfaillie K (2004) Perception of biological motion: a stimulus set of human point-light actions. Behavior Research Methods, Instruments, \& Computers 36: 625-629. [AQ: 15]

Weigelt S, Koldewyn K and Kanwisher N (2012) Face identity recognition in autism spectrum disorders: a review of behavioral studies. Neuroscience and Biobehavioral Reviews 36: 1060-1084. 